\title{
GENERATING FUNCTIONS AND GENERALIZED DEDEKIND SUMS
}

\author{
IRA M. GESSEL \\ Department of Mathematics \\ Brandeis University \\ Waltham, MA 02254-9110 \\ gessel@math.brandeis.edu
}

Submitted: August 31, 1996; Accepted: October 1, 1996

Dedicated to Herb Wilf, in honor of his 65th birthday

\begin{abstract}
We study sums of the form $\sum_{\zeta} R(\zeta)$, where $R$ is a rational function and the sum is over all $n$th roots of unity $\zeta$ (often with $\zeta=1$ excluded). We call these generalized Dedekind sums, since the most well-known sums of this form are Dedekind sums. We discuss three methods for evaluating such sums: The method of factorization applies if we have an explicit formula for $\prod_{\zeta}(1-x R(\zeta))$. Multisection can be used to evaluate some simple, but important sums. Finally, the method of partial fractions reduces the evaluation of arbitrary generalized Dedekind sums to those of a very simple form.
\end{abstract}

\section{Introduction.}

Given a rational function $R(x)$, we consider the problem of evaluating the sum

$$
\sum_{\zeta} R(\zeta)
$$

over all $n$th roots of unity $\zeta$ (often with $\zeta=1$ excluded.) Such problems arise in several areas of mathematics, such as number theory and topology, and this work was originally motivated by a question from Larry Smith [9] regarding sums of this form that arose in his work on stable homotopy theory [8].

Although there is a large literature on special instances of such sums, there does not seem to have been any discussion of the general problem. Since the special cases that have been studied are usually called Dedekind sums, we call the sums considered here generalized Dedekind sums. For a comprehensive account of the classical theory of Dedekind sums, see Rademacher and Grosswald[7] An elegant

1991 Mathematics Subject Classification. Primary 11F20, Secondary 05A15.

This work is partially supported by NSF grant DMS-9622456. 
treatment of an important generalization of the classical Dedekind sum has been given by Zagier [11].

In this paper we discuss three methods, all using generating functions, for studying such sums. The first method is factorization: If we have an "explicit" formula for the product $P(x)=\prod_{j}\left(1-\alpha_{j} x\right)$, then we can use it to study the sums $\sum_{j} \alpha_{j}^{k}$. We apply this in the case in which $\alpha_{j}$ is $R\left(\zeta_{j}\right)$ for some rational function $R$, where $\zeta_{j}$ is an $n$th root of unity.

The second method is multisection: If $R(x)=\sum_{k} r_{k} x^{k}$ then $\sum_{\zeta^{n}=1} R(\zeta x)=$ $n \sum_{k} r_{n k} x^{n k}$. If $R$ is rational and we have an explicit formula for $\sum_{k} r_{n k} x^{n k}$, then we have evaluated $\sum_{\zeta^{n}=1} R(\zeta x)$, and we can set $x=1$ (sometimes after subtracting the $\zeta=1$ term) to evaluate $\sum_{\zeta^{n}=1} R(\zeta)$ or $\sum_{\substack{\zeta^{n}=1 \\ \zeta \neq 1}} R(\zeta)$.

The third, and most powerful, method is partial fractions: Since any rational function is a linear combination of rational functions of the form $(x-\alpha)^{-i}$, the general problem may be reduced to the case of this particular form, which can be solved by either of the first two methods or by a further application of partial fractions. Partial fractions can also be used to derive "reciprocity theorems" which are important in the theory of classical Dedekind sums.

\section{Factorization.}

Let $P(x)$ be a polynomial and suppose that

$$
P(x)=\prod_{j}\left(1-\alpha_{j} x\right)
$$

Then

$$
-\log P(x)=\sum_{k=1}^{\infty}\left(\sum_{j} \alpha_{j}^{k}\right) \frac{x^{k}}{k}
$$

Thus if for some rational function $R, \alpha_{j}$ is $R\left(\zeta_{j}\right)$, where $\zeta_{j}$ is a root of unity, and if we know $P(x)$ explicitly, then we have a generating function for $\sum_{j} R\left(\zeta_{j}\right)^{k}$.

The simplest interesting example comes from

$$
z^{n}-1=\prod_{\zeta^{n}=1}(z-\zeta)
$$

Setting $z=x+\alpha$ in $(2.2)$ gives

$$
(x+\alpha)^{n}-1=\prod_{\zeta^{n}=1}(x-(\zeta-\alpha)) .
$$

Dividing each side by its constant term we get

$$
\frac{(\alpha+x)^{n}-1}{\alpha^{n}-1}=\prod_{\zeta^{n}=1}\left(1-\frac{x}{\zeta-\alpha}\right) .
$$


Then applying (2.1), we have

$$
\log \frac{\alpha^{n}-1}{(\alpha+x)^{n}-1}=\sum_{k=1}^{\infty}\left(\sum_{\zeta^{n}=1} \frac{1}{(\zeta-\alpha)^{k}}\right) \frac{x^{k}}{k}
$$

Extracting the coefficients of $x$ and $x^{2}$ in (2.3) gives

$$
\begin{aligned}
\sum_{\zeta^{n}=1} \frac{1}{\zeta-\alpha} & =-n \frac{\alpha^{n-1}}{\alpha^{n}-1} \\
\sum_{\zeta^{n}=1} \frac{1}{(\zeta-\alpha)^{2}} & =n \frac{\alpha^{n-2}\left(\alpha^{n}+n-1\right)}{\left(\alpha^{n}-1\right)^{2}}
\end{aligned}
$$

Similarly, we may start from

$$
\frac{z^{n}-1}{z-1}=\prod_{\substack{\zeta^{n}=1 \\ \zeta \neq 1}}(z-\zeta)
$$

Setting $z=x+1$ in (2.6) gives

$$
\frac{(1+x)^{n}-1}{x}=\prod_{\substack{\zeta n=1 \\ \zeta \neq 1}}(x-(\zeta-1)) .
$$

Dividing each side by its constant term we get

$$
\frac{(1+x)^{n}-1}{n x}=\prod_{\substack{\zeta n=1 \\ \zeta \neq 1}}\left(1-\frac{x}{\zeta-1}\right)
$$

Now let

$$
g_{k}(n)=\sum_{\substack{\zeta^{n}=1 \\ \zeta \neq 1}}(\zeta-1)^{-k}
$$

Using the facts that if $\zeta=e^{2 \pi i j / n}$, where $i=\sqrt{-1}$, then

$$
\frac{1}{(\zeta-1)^{k}}=\frac{\zeta^{-k / 2}}{\left(\zeta^{1 / 2}-\zeta^{-1 / 2}\right)^{k}}=\left(\frac{1}{2 i}\right)^{k} \frac{\cos \frac{\pi j k}{n}-i \sin \frac{\pi j k}{n}}{\sin ^{k} \frac{\pi j}{n}}
$$

and that $g_{k}(n)$ is real, we obtain trigonometric formulas for $g_{k}(n)$ : If $k$ is even,

$$
g_{k}(n)=\frac{(-1)^{k / 2}}{2^{k}} \sum_{j=1}^{n-1} \cos \frac{\pi j k}{n} \csc ^{k} \frac{\pi j}{n}
$$


and if $k$ is odd,

By $(2.1)$

$$
g_{k}(n)=\frac{(-1)^{(k-1) / 2}}{2^{k}} \sum_{j=1}^{n-1} \sin \frac{\pi j k}{n} \csc ^{k} \frac{\pi j}{n} .
$$

$$
\sum_{k=1}^{\infty} g_{k}(n) \frac{x^{k}}{k}=\log \frac{n x}{(1+x)^{n}-1}
$$

From (2.7), we can easily compute the first few values of $g_{k}(n)$ :

$$
\begin{aligned}
& g_{1}(n)=-(n-1) / 2 \\
& g_{2}(n)=-(n-1)(n-5) / 12 \\
& g_{3}(n)=(n-1)(n-3) / 8 \\
& g_{4}(n)=(n-1)\left(n^{3}+n^{2}-109 n+251\right) / 720 \\
& g_{5}(n)=-(n-1)(n-5)\left(n^{2}+6 n-19\right) / 288 \\
& g_{6}(n)=-(n-1)\left(2 n^{5}+2 n^{4}-355 n^{3}-355 n^{2}+11153 n-19087\right) / 60480
\end{aligned}
$$

The problem of showing that $g_{k}(n)=\sum_{\substack{\zeta^{n}=1 \\ \zeta \neq 1}}(\zeta-1)^{-k}$ is a polynomial in $n$ of degree at most $k$ with rational coefficients was proposed by Duran [5]. This result follows easily from (2.7), but we can say much more about these pbynomials. by

First we recall that the unsigned Stirling numbers of the first kind $\left[\begin{array}{l}n \\ k\end{array}\right]$ are defined

$$
\frac{(\log (1+x))^{k}}{k !}=\sum_{n=k}^{\infty}(-1)^{n-k}\left[\begin{array}{l}
n \\
k
\end{array}\right] \frac{x^{n}}{n !}
$$

and the Bernoulli numbers $B_{n}$ are defined by

$$
\frac{x}{e^{x}-1}=\sum_{n=0}^{\infty} B_{n} \frac{x^{n}}{n !}
$$

It is well known that $B_{1}=-1 / 2$, and for $n>1 B_{n}$ is zero if and only if $n$ is odd.

Theorem 2.1. For $k \geq 1$,

$$
g_{k}(n)=(-1)^{k} \frac{n-1}{2}-\frac{1}{(k-1) !} \sum_{j=2}^{k}(-1)^{k-j}\left[\begin{array}{c}
k \\
j
\end{array}\right] \frac{B_{j}}{j}\left(n^{j}-1\right)
$$

Proof. Let us set $x=e^{y}-1$, so that $y=\log (1+x)$. Then by $(2.7)$,

$$
\sum_{k=1}^{\infty} g_{k}(n) \frac{x^{k}}{k}=-\log \frac{e^{n y}-1}{n\left(e^{y}-1\right)}=\log \frac{n y}{e^{n y}-1}-\log \frac{y}{e^{y}-1}
$$


Since

$$
\frac{d}{d u} \log \frac{u}{e^{u}-1}=\frac{1}{u}\left(-\frac{u}{e^{u}-1}+1-u\right)=-\frac{1}{2}-\sum_{j=1}^{\infty} \frac{B_{j+1}}{j+1} \frac{u^{j}}{j !}
$$

we have

$$
\begin{aligned}
\sum_{k=1}^{\infty} g_{k}(n) \frac{x^{k}}{k} & =-\frac{n y}{2}-\sum_{j=2}^{\infty} \frac{B_{j}}{j} \frac{(n y)^{j}}{j !}+\frac{y}{2}+\sum_{j=2}^{\infty} \frac{B_{j}}{j} \frac{y^{j}}{j !} \\
& =-\frac{(n-1)}{2} y-\sum_{j=2}^{\infty} \frac{B_{j}}{j}\left(n^{j}-1\right) \frac{y^{j}}{j !} .
\end{aligned}
$$

Now

$$
\frac{y^{j}}{j !}=\sum_{k=j}^{\infty}(-1)^{k-j}\left[\begin{array}{c}
k \\
j
\end{array}\right] \frac{x^{k}}{k !}
$$

so it follows from (2.8) that

$$
g_{k}(n)=(-1)^{k} \frac{n-1}{2}-\frac{1}{(k-1) !} \sum_{j=2}^{k}(-1)^{k-j}\left[\begin{array}{l}
k \\
j
\end{array}\right] \frac{B_{j}}{j}\left(n^{j}-1\right) .
$$

By taking $n \rightarrow 0$ in $(2.7)$, we find that

$$
\sum_{k=1}^{\infty} g_{k}(0) \frac{x^{k}}{k}=\log \frac{x}{\log (1+x)} .
$$

Differentiating this formula with respect to $x$ then multiplying by $x$, we obtain

$$
\sum_{k=1}^{\infty} g_{k}(0) x^{k}=1-\frac{x}{(1+x) \log (1+x)} .
$$

Thus $g_{k}(0)=-N_{k} / k$ !, where the Nörlund numbers $N_{k}$ are defined by

$$
\frac{x}{(1+x) \log (1+x)}=\sum_{k=0}^{\infty} N_{k} \frac{x^{k}}{k !}
$$

(see Howard [6]). So the formula for $g_{k}(n)$ given by Theorem 2.1 nay be restated as

$$
g_{k}(n)=-\frac{N_{k}}{k !}+(-1)^{k} \frac{n}{2}-\frac{1}{(k-1) !} \sum_{j=2}^{k}(-1)^{k-j}\left[\begin{array}{c}
k \\
j
\end{array}\right] \frac{B_{j}}{j} n^{j} .
$$

Since $\left[\begin{array}{l}k \\ k\end{array}\right]=1$ and $\left[\begin{array}{c}k \\ k-1\end{array}\right]=\left(\begin{array}{c}k \\ 2\end{array}\right)$, for $k \geq 2$ the leading term of $g_{k}(n)$ is $-\left(B_{k} / k !\right) n^{k}$ for $k$ even and $(k / 2(k-1) !) B_{k-1} n^{k-1}$ for $k$ odd. Moreover, $g_{k}(n)-(-1)^{k} n / 2$ contains only even powers of $n$.

It is clear that $g_{k}(1)=0$ for every $k$, so $g_{k}(n)$ is divisible by $n-1$. Empirical evidence suggests that other than the fact that $g_{2}(n)$ is divisible by $n-5$, the only other factorization of the polynomials $g_{k}(n)$ over the rationals is given by the following result. 
Proposition 2.2. If $k$ is odd, then as a polynomial in $n, g_{k}(n)$ is divisible by $n-d$ for every positive divisor $d$ of $k$.

Proof. We shall show that if $d$ is a positive divisor of $k$ then $g_{k}(d)=0$.

Let $\xi$ be a primitive $d$ th root of unity, where $d$ is odd. We want to show that if $q$ is odd then

$$
\sum_{j=1}^{d-1}\left(\xi^{j}-1\right)^{-d q}=0
$$

Let $A$ be the sum in question. Then for any integer $l$,

$$
A=\xi^{-d q l} A=\sum_{j=1}^{d-1}\left(\xi^{j+l}-\xi^{l}\right)^{-d q} .
$$

Thus

$$
d A=\sum_{l=0}^{d-1} \xi^{-d q l} A=\sum_{\substack{0 \leq l, m \leq d-1 \\ l \neq m}}\left(\xi^{m}-\xi^{l}\right)^{-d q} .
$$

Interchanging $m$ and $l$ in the last sum multiplies each term by $(-1)^{d q}=-1$ and also permutes the terms in the sum. Thus $d A=-d A$, so $A=0$.

As another example of the method of factorization, set $z=(1+x) /(1-x)$ in (2.6). Then we have

$$
\frac{\left(\frac{1+x}{1-x}\right)^{n}-1}{2 x /(1-x)}=\prod_{\substack{\zeta n=1 \\ \zeta \neq 1}}\left(\frac{1+x}{1-x}-\zeta\right) .
$$

Multiplying both sides by $(1-x)^{n-1}$, dividing each side by its constant term, and simplifying, we get

$$
\frac{(1+x)^{n}-(1-x)^{n}}{2 n x}=\prod_{\substack{\zeta n=1 \\ \zeta \neq 1}}\left(1-\frac{\zeta+1}{\zeta-1} x\right) .
$$

As before, we get

$$
\log \frac{2 n x}{(1+x)^{n}-(1-x)^{n}}=\sum_{k=1}^{\infty}\left[\sum_{\substack{\zeta=1 \\ \zeta \neq 1}}\left(\frac{\zeta+1}{\zeta-1}\right)^{k}\right] \frac{x^{k}}{k} .
$$

If we set

$$
q_{k}(n)=\sum_{\substack{\zeta^{n}=1 \\ \zeta \neq 1}}\left(\frac{\zeta+1}{\zeta-1}\right)^{k}
$$


then $q_{k}(n)$ is 0 for $k$ odd, and the first few values for even $k$, computed from this generating function, are

$$
\begin{aligned}
& q_{2}(n)=-\frac{1}{3}(n-1)(n-2) \\
& q_{4}(n)=\frac{1}{45}(n-1)(n-2)\left(n^{2}+3 n-13\right) \\
& q_{6}(n)=-\frac{1}{945}(n-1)(n-2)\left(2 n^{4}+6 n^{3}-28 n^{2}-96 n+251\right)
\end{aligned}
$$

We also have a simple trigonometric formula:

$$
q_{k}(n)=(-1)^{k / 2} \sum_{j=1}^{n-1} \cot ^{k} \frac{\pi j}{n}, \quad k \text { even }
$$

It may be noted that $q_{k}(n)$ is a special case of the "higher-dimensional Dedekind sums" studied by Zagier [11]

A more difficult application of factorization is a result of Stanley [10]:

Theorem 2.3. Let

$$
S_{k}(n)=\sum_{\substack{\zeta^{n}=1 \\ \zeta \neq 1}}|1-\zeta|^{-2 k}
$$

Then

$$
\sum_{k=1}^{\infty} 4^{k} S_{k}(n) x^{2 k}=1-\frac{n x \cot \left(n \sin ^{-1} x\right)}{\sqrt{1-x^{2}}} .
$$

Proof. First note that since $|1-\zeta|^{-2}=1 /(1-\zeta)\left(1-\zeta^{-1}\right)=-\zeta /(1-\zeta)^{2}$, we have

$$
S_{k}(n)=\sum_{\substack{\zeta^{n}=1 \\ \zeta \neq 1}}|1-\zeta|^{-2 k}=\sum_{\substack{\zeta^{n}=1 \\ \zeta \neq 1}} \frac{(-\zeta)^{k}}{(1-\zeta)^{2 k}}=\sum_{j=1}^{n-1}\left(\frac{1}{2} \csc \frac{\pi j}{n}\right)^{2 k} .
$$

Now since

$$
\frac{d}{d x} \log \sin \left(n \sin ^{-1} x\right)=\frac{n \cot \left(n \sin ^{-1} x\right)}{\sqrt{1-x^{2}}}
$$

we have

$$
1-\frac{n x \cot \left(n \sin ^{-1} x\right)}{\sqrt{1-x^{2}}}=-x \frac{d}{d x}\left[\log \frac{\sin \left(n \sin ^{-1} x\right)}{x}\right] .
$$

So to prove Stanley's formula we must show that

$$
\log \frac{\sin \left(n \sin ^{-1} x\right)}{C x}=-\sum_{k=1}^{\infty} 4^{k} S_{k}(n) \frac{x^{2 k}}{2 k}=-\sum_{k=1}^{\infty}\left(\sum_{j=1}^{n-1} \csc ^{2 k} \frac{\pi j}{n}\right) \frac{x^{2 k}}{2 k}
$$


where $\log C$ is an appropriate constant of integration. Since $\sin \left(n \sin ^{-1} x\right) / C x$ must have constant term $1, C$ must be $n$. Then multiplying both sides of (2.10) by 2 and exponentiating, we see that the identity to be proved is

$$
\left(\frac{\sin \left(n \sin ^{-1} x\right)}{n x}\right)^{2}=\prod_{j=1}^{n-1}\left(1-x^{2} \csc ^{2} \frac{\pi j}{n}\right) .
$$

The right side of (2.11) is a polynomial in $x$ whose degree, constant terms, and roots are easily determined; so it is sufficient to show that these are the same for the left side. There is a complication due to the multiplicity 2 of most of the roots, which leads us to consider separately the cases $n$ even and $n$ odd.

First we examine the roots of the right side of (2.11). The right side of (2.11) vanishes for

$$
x= \pm \sin \frac{\pi j}{n}, \quad j=1,2, \ldots, n-1
$$

but since $\sin \frac{\pi j}{n}=\sin \frac{\pi(n-j)}{n}$, each $\pm \sin \frac{\pi j}{n}$ with $1 \leq j<n / 2$ appears twice as a root. Moreover, if $n$ is even then each of $\pm \sin \frac{\pi}{2}= \pm 1$ appears once as a root. This takes care of all $2 n-2$ roots.

Next we consider the left side of (2.10). It is easy to prove (e.g., by induction) that if $n$ is odd then $\sin n \theta$ is a polytromial of degree $n$ in $\sin \theta$ and if $n$ is even then $\sin n \theta / \cos \theta$ is a polynomial of degree $n-1$ in $\sin \theta$.

Thus

$$
\sin \left(n \sin ^{-1} x\right)= \begin{cases}P_{n}(x), & \text { if } n \text { is odd } \\ \sqrt{1-x^{2}} Q_{n-1}(x), & \text { if } n \text { is even }\end{cases}
$$

where $P_{m}(x)$ and $Q_{m}(x)$ are polynomials in $x$ of degree $m .^{1}$ If $x= \pm \sin \frac{\pi j}{n}$ for some integer $j$, then $\sin \left(n \sin ^{-1} x\right)=0$. Thus if $n$ is odd,

$$
\left(\frac{\sin \left(n \sin ^{-1} x\right)}{n x}\right)^{2}
$$

is a polynomial of degree $2 n-2$ with constant term 1 and with roots $\pm \sin \frac{\pi j}{n}$ for $j=1,2, \ldots,(n-1) / 2$, each with multiplicity (at least) 2 ; if $n$ is even then $(2.12)$ is a polynomial of degree $2 n-2$ with constant term 1 and with roots $\pm \sin \frac{\pi j}{n}$ for $j=1,2, \ldots, n / 2-1$, each with multiplicity (at least) 2 and with roots \pm 1 each of multiplicity (at least) 1. These facts are sufficient to establish (2.11), and thus Stanley's formula.

It is also possible to give an explicit formula, analogous to Theorem 2.1, for the coefficients of $S_{2 k}(n)$ in terms of Bernoulli numbers and central factorial mumbers.

\footnotetext{
${ }^{1}$ It can be shown that for $n$ odd, $P_{n}(x)=(-1)^{(n-1) / 2} T_{n}(x)$ and for $n$ even, $Q_{n-1}(x)=$ $(-1)^{(n / 2)-1} U_{n-1}(x)$, where $T_{n}(x)$ and $U_{n-1}(x)$ are the Chebyshev polynomials of the first and second kinds, defined by $\cos n \theta=T_{n}(\cos \theta)$ and $\sin n \theta=U_{n-1}(\cos \theta) \sin \theta$.
} 


\section{Multisection.}

Let $R(x)$ be a rational function of $x$. Then $R$ has a Laurent series expansion

$$
R(x)=\sum_{i=N}^{\infty} r_{i} x^{i}
$$

By $n$-section of $R(x)$ we mean the extraction of the sum of the terms $r_{i} x^{i}$ in which $i$ is divisible by $n$. It is well-known (and easy to prove) that

$$
\sum_{\zeta^{n}=1} R(\zeta x)=n \sum_{k} r_{n k} x^{n k}
$$

In some cases, we have an explicit formula for $r_{i}$ that we can use, with the help of (3.1), to evaluate $\sum_{\zeta^{n}=1} R(\zeta x)$.

We note that the method of multisection is closely related to the invariant theory method used by Stanley [10] to evaluate some generalized Dedekind sums.

As a simple example of this approach, take $R(x)=x /\left(1-x-x^{2}\right)=\sum_{i=0}^{\infty} F_{i} x^{i}$, the generating function for the Fibonacci numbers. It is well known that $F_{i}=$ $\left(\alpha^{i}-\beta^{i}\right) /(\alpha-\beta)$, where $\alpha, \beta=(1 \pm \sqrt{5}) / 2$, so we have

$$
\begin{aligned}
\sum_{k=0}^{\infty} F_{n k} x^{n k} & =\sum_{k=0}^{\infty} \frac{\alpha^{n k}-\beta^{n k}}{\alpha-\beta} x^{n k} \\
& =\frac{1}{\alpha-\beta}\left(\frac{1}{1-\alpha^{n} x^{n}}-\frac{1}{1-\beta^{n} x^{n}}\right) \\
& =\frac{1}{\alpha-\beta}\left(\frac{\left(\alpha^{n}-\beta^{n}\right) x^{n}}{1-\left(\alpha^{n}+\beta^{n}\right) x^{n}+(\alpha \beta)^{n} x^{2 n}}\right) \\
& =\frac{F_{n} x^{n}}{1-L_{n} x^{n}+(-1)^{n} x^{2 n}}
\end{aligned}
$$

where $L_{n}=\alpha^{n}+\beta^{n}$ is the $n$th Lucas number. Thus

$$
\sum_{\zeta^{n}=1} \frac{\zeta x}{1-\zeta x-(\zeta x)^{2}}=\frac{n F_{n} x^{n}}{1-L_{n} x^{n}+(-1)^{n} x^{2 n}} .
$$

Although we proved (3.2) under the assumption that $x$ is an indeterminate, since both sides are rational functions of $x,(3.2)$ must also hold as an identity of rational functions. Thus we may set $x=1$ in (3.2) to obtain

$$
\sum_{\zeta^{n}=1} \frac{\zeta}{1-\zeta-\zeta^{2}}=\frac{n F_{n}}{1+(-1)^{n}-L_{n}} .
$$

Note that as a consequence of (3.3) we have the curious formula

$$
\lim _{n \rightarrow \infty} \frac{1}{n} \sum_{\zeta^{n}=1} \frac{1}{1-\zeta-\zeta^{2}}=-\frac{1}{\sqrt{5}}
$$

Next we apply multisection to prove a simple but fundamental and important result. 
Theorem 3.1. Let $r$ be an integer with $1 \leq r \leq n$. Then if $x \neq y$,

$$
\sum_{\zeta^{n}=1} \frac{\zeta^{r}}{x-y \zeta}=n \frac{x^{r-1} y^{n-r}}{x^{n}-y^{n}}
$$

Proof. Since both sides are homogeneous of degree -1 in $x$ and $y$, it is sufficient to prove the case in which $x=1$. Moreover, since both sides are rational functions of $x$ and $y$, we may assume that $y$ is an indeterminate, so that the left side can be expanded as a power series in $y$. Then since $n$-secting $y^{r} /(1-y)=y^{r}+y^{r+1}+\cdots$ yields $y^{n}+y^{2 n}+\cdots=y^{n} /\left(1-y^{n}\right)$, we obtain

$$
\sum_{\zeta^{n}=1} \frac{(y \zeta)^{r}}{1-y \zeta}=\frac{n y^{n}}{1-y^{n}}
$$

and this is equivalent to the formula to be proved.

Note that since the sum on the left side of (3.4) depends only on the congruence class of $r$ modulo $n$, Theorem 3.1 can be used to evaluate this sum for all $r$. In particular, the case $r=n$ is equivalent to $(2.4)$.

Corollary 3.2. If $1 \leq r \leq n$ then

$$
\sum_{\substack{\zeta^{n}=1 \\ \zeta \neq 1}} \frac{\zeta^{r}}{1-\zeta}=r-\frac{n-1}{2}
$$

Proof. By Theorem 3.1,

$$
\sum_{\substack{\zeta n=1 \\ \zeta \neq 1}} \frac{\zeta^{r}}{1-y \zeta}=n \frac{y^{n-r}}{1-y^{n}}-\frac{1}{1-y}
$$

The corollary follows by taking the limit as $y \rightarrow 1$.

Our next corollary generalizes (2.3) and (2.7).

Corollary 3.3. If $1 \leq r \leq n$ then

$$
\sum_{k=1}^{\infty} u^{k-1} \sum_{\zeta^{n}=1} \frac{\zeta^{r}}{(x-y \zeta)^{k}}=n \frac{(x-u)^{r-1} y^{n-r}}{(x-u)^{n}-y^{n}}
$$

and

$$
\sum_{k=1}^{\infty} u^{k-1} \sum_{\substack{\zeta^{n}=1 \\ \zeta \neq 1}} \frac{\zeta^{r}}{(1-\zeta)^{k}}=\frac{1}{u}+n \frac{(1-u)^{r-1}}{(1-u)^{n}-1}
$$


Proof. We have

$$
\begin{aligned}
\sum_{k=1}^{\infty} u^{k-1} \sum_{\zeta^{n}=1} \frac{\zeta^{r}}{(x-y \zeta)^{k}} & =\sum_{\zeta^{n}=1} \zeta^{r} \sum_{k=1}^{\infty} \frac{u^{k-1}}{(x-y \zeta)^{k}}=\sum_{\zeta^{n}=1} \frac{\zeta^{r}}{x-u-y \zeta} \\
& =n \frac{(x-u)^{n-r^{\prime}-1} y^{r^{\prime}}}{(x-u)^{n}-y^{n}}
\end{aligned}
$$

which proves (3.6). To prove (3.7), subtract from (3.6) its specialization at $n=1$ (and $r=1$ ), and then set $x=y=1$.

In particular, it follows from Corollary 3.3 that

$$
\sum_{\zeta^{n}=1} \frac{\zeta^{r}}{(x-y \zeta)^{2}}=n \frac{x^{r-2} y^{n-r}\left((n+1-r) x^{n}+(r-1) y^{n}\right)}{\left(x^{n}-y^{n}\right)^{2}},
$$

which can also be obtained by differentiating (3.4) with respect to $x$. The case $r=n$ of $(3.8)$ is equivalent to $(2.5)$.

If we take $r=1$ in $(3.7)$ we get

$$
\sum_{k=1}^{\infty} u^{k-1} \sum_{\substack{\zeta^{n}=1 \\ \zeta \neq 1}} \frac{\zeta}{(1-\zeta)^{k}}=\frac{1}{u}+\frac{n}{(1-u)^{n}-1} .
$$

L. Carlitz $[3,4]$ has studied the "degenerate Bernoulli numbers" $\beta_{k}(\lambda)$ defined by

$$
\sum_{k=0}^{\infty} \beta_{k}(\lambda) \frac{u^{k}}{k !}=\frac{u}{(1+\lambda u)^{1 / \lambda}-1}
$$

Comparing (3.9) with (3.10), we see that

$$
\sum_{\substack{\zeta^{n}=1 \\ \zeta \neq 1}} \frac{\zeta}{(1-\zeta)^{k}}=\frac{(-1)^{k-1}}{k !} n^{k} \beta_{k}(1 / n) .
$$

More generally, Carlitz [4, Section 5] considered "degenerate Bernoulli polynomials" $\beta_{k}(\lambda, z)$ defined by

$$
\sum_{k=0}^{\infty} \beta_{k}(\lambda, z) \frac{u^{k}}{k !}=\frac{u(1+\lambda u)^{z / \lambda}}{(1+\lambda u)^{1 / \lambda}-1} .
$$

Taking $r=s+1$ in (3.7), where $0 \leq s \leq n-1$, and comparing with (3.12) gives the following result:

Corollary 3.4. For $0 \leq s \leq n-1$,

$$
\sum_{\substack{\zeta^{n}=1 \\ \zeta \neq 1}} \frac{\zeta^{s+1}}{(1-\zeta)^{k}}=\frac{(-1)^{k-1}}{k !} n^{k} \beta_{k}(1 / n, s / n)
$$




\section{Partial Fractions.}

We have already seen, at least implicitly, some examples of partial fraction expansions: the logarithmic derivatives of the factorizations in section 2 are partial fraction expansions, and Theorem 3.1 may be viewed as a partial fraction expansion. Since any rational function of $\zeta$ can be expressed as polynomial plus a linear combination of rational functions of the form $(1-\alpha \zeta)^{-k}$, and we know how to evaluate $\sum_{\zeta^{n}=1}(1-\alpha \zeta)^{-k}$, we can in principle evaluate any generalized Dedekind sum by partial fractions.

As a first application of this method, we consider an American Mathematical Monthly problem proposed by P. E. Bjørstad and H. Fettis [1] and solved by H.-J. Seiffert: to find a "closed algebraic expression" for the sum

$$
S_{N}=\sum_{k=1}^{N-1} \frac{\sin ^{2} \frac{k \pi}{N}}{\left(1-2 a \cos \frac{k \pi}{N}+a^{2}\right)^{2}} .
$$

We give a simpler derivation of Seiffert's formula:

\section{Proposition 4.1.}

$$
S_{N}=\frac{N}{2\left(1-a^{2 N}\right)}\left(\frac{1+a^{2 N-2}}{1-a^{2}}-2 N \frac{a^{2 N-2}}{1-a^{2 N}}\right) .
$$

Proof. To evaluate $S_{N}$, we first express it as a generalized Dedekind sum. We note that the summand is an even function of $k$ that vanishes when $k$ is divisible by $N$. Thus

$$
S_{N}=\frac{1}{2} \sum_{k=-N}^{N-1} \frac{\sin ^{2} \frac{k \pi}{N}}{\left(1-2 a \cos \frac{k \pi}{N}+a^{2}\right)^{2}} .
$$

Expressing the trigonometric functions in terms of roots of unity, we have

$$
S_{N}=-\frac{1}{8} \sum_{\zeta^{2 N}=1} \frac{\left(\zeta-\zeta^{-1}\right)^{2}}{\left[(1-a \zeta)\left(1-a \zeta^{-1}\right)\right]^{2}} .
$$

Now for any integer $n$, let us set

$$
T_{n}=\sum_{\zeta^{n}=1} \frac{\left(\zeta-\zeta^{-1}\right)^{2}}{\left[(1-a \zeta)\left(1-a \zeta^{-1}\right)\right]^{2}},
$$

so that $S_{N}=-T_{2 N} / 8$.

We have the partial fraction expansion

$$
\begin{aligned}
\frac{\left(\zeta-\zeta^{-1}\right)^{2}}{\left[(1-a \zeta)\left(1-a \zeta^{-1}\right)\right]^{2}}=\frac{1}{a^{2}} & -\frac{2}{a^{2}\left(1-a^{2}\right)(1-a \zeta)} \\
& +\frac{1}{a^{2}(1-a \zeta)^{2}}+\frac{2}{\left(1-a^{2}\right)(1-\zeta / a)}+\frac{1}{a^{2}(1-\zeta / a)^{2}} .
\end{aligned}
$$

Summing over $\zeta^{n}-1$, applying formulas (3.4) and (3.8), and simplifying yields

$$
T_{n}=-\frac{2 n}{1-a^{n}}\left(\frac{1+a^{n-2}}{1-a^{2}}-n \frac{a^{n-2}}{1-a^{n}}\right)
$$

and the theorem follows.

We now give one of the many possible generalizations of Proposition 4.1. 
Theorem 4.2. Let

$$
P_{n}(k, l, r)=\sum_{\zeta^{n}=1} \frac{\zeta^{r}}{(1-a \zeta)^{k}\left(1-a \zeta^{-1}\right)^{l}}
$$

Then if $1 \leq r \leq n-1$,

$$
\begin{aligned}
\sum_{k, l=0}^{\infty} P_{n}(k, l, r) x^{k} y^{l}=\frac{n}{(1-x)(1-y)-a^{2}}( & x\left(1-x-a^{2}\right) \frac{(1-x)^{r-1} a^{n-r}}{(1-x)^{n}-a^{n}} \\
& \left.+y\left(1-y-a^{2}\right) \frac{(1-y)^{n-r-1} a^{r}}{(1-y)^{n}-a^{n}}\right)
\end{aligned}
$$

and

$$
\begin{array}{r}
\sum_{k, l=0}^{\infty} P_{n}(k, l, 0) x^{k} y^{l}=n+\frac{n}{(1-x)(1-y)-a^{2}}\left(x\left(1-x-a^{2}\right) \frac{(1-x)^{n-1}}{(1-x)^{n}-a^{n}}\right. \\
\left.+y\left(1-y-a^{2}\right) \frac{(1-y)^{n-1}}{(1-y)^{n}-a^{n}}-x y\right)
\end{array}
$$

Proof. We have

$$
\begin{aligned}
\sum_{k, l=0}^{\infty} P_{n}(k, l, r) x^{k} y^{l} & =\sum_{k, l=0}^{\infty} x^{k} y^{l} \sum_{\zeta^{n}=1} \frac{\zeta^{r}}{(1-a \zeta)^{k}\left(1-a \zeta^{-1}\right)^{l}} \\
& =\sum_{\zeta^{n}=1} \zeta^{r} \sum_{k, l} \frac{x^{k} y^{l}}{(1-a \zeta)^{k}\left(1-a \zeta^{-1}\right)^{l}} .
\end{aligned}
$$

Then

$$
\begin{aligned}
& \sum_{k, l=0}^{\infty} \frac{x^{k} y^{l}}{(1-a \zeta)^{k}\left(1-a \zeta^{-1}\right)^{l}}=\left(1-\frac{x}{1-a \zeta}\right)^{-1}\left(1-\frac{y}{1-a \zeta^{-1}}\right)^{-1} \\
& =1+\frac{1}{(1-x)(1-y)-a^{2}}\left(\frac{x\left(1-x-a^{2}\right)}{1-x-a \zeta}+\frac{y\left(1-y-a^{2}\right)}{1-y-a \zeta^{-1}}-x y\right)
\end{aligned}
$$

Now multiply each side by $\zeta^{r}$ and sum over all $\zeta$ with $\zeta^{n}=1$. The sum can be evaluated by Theorem 3.1, using the fact that

$$
\sum_{\zeta^{n}=1} \frac{\zeta^{r}}{1-y-a \zeta^{-1}}=\sum_{\zeta^{n}=1} \frac{\zeta^{-r}}{1-y-a \zeta}=\sum_{\zeta^{n}=1} \frac{\zeta^{n-r}}{1-y-a \zeta}
$$

Next we show how Stanley's formula Theorem 2.3) can be proved by partial fractions. Although the proof we gave earlier suggested that this result depends on 
a very special factorization, the proof we give now shows that Stanley's formula is an instance (though an especially nice one) of a much more general phenomenon.

To prove Stanley's formula we must evaluate

$$
\sum_{k=1}^{\infty} 4^{k} S_{k}(n) x^{2 k}=\sum_{k=1}^{\infty}(2 x)^{2 k} \sum_{\substack{\zeta n=1 \\ \zeta \neq 1}} \frac{(-\zeta)^{k}}{(1-\zeta)^{2 k}}=-\sum_{\substack{\zeta n=1 \\ \zeta \neq 1}} \frac{4 x^{2} \zeta}{(1-\zeta)^{2}+4 x^{2} \zeta} .
$$

We proceed by expanding

$$
-\frac{4 x^{2} \zeta}{(1-\zeta)^{2}+4 x^{2} \zeta}
$$

in partial fractions. Factoring the denominator of (4.1), we find that

$$
(1-\zeta)^{2}+4 x^{2} \zeta=(1-A \zeta)(1-B \zeta)
$$

where $A=1-2 x^{2}+2 i x \sqrt{1-x^{2}}$ and $B=1-2 x^{2}-2 i x \sqrt{1-x^{2}}$, and we obtain the partial fraction expansion

$$
-\frac{4 x^{2} \zeta}{(1-\zeta)^{2}+4 x^{2} \zeta}=\frac{i x}{\sqrt{1-x^{2}}}\left(\frac{1}{1-A \zeta}-\frac{1}{1-B \zeta}\right) .
$$

Summing over $\zeta^{n}=1$ yields

$$
-\sum_{\zeta^{n}=1} \frac{4 x^{2} \zeta}{(1-\zeta)^{2}+4 x^{2} \zeta}=\frac{n i x}{\sqrt{1-x^{2}}}\left(\frac{1}{1-A^{n}}-\frac{1}{1-B^{n}}\right) .
$$

Since $A B=1$, we have

$$
\frac{1}{1-A^{n}}-\frac{1}{1-B^{n}}=\frac{1}{1-A^{n}}-\frac{A^{n}}{A^{n}-1}=\frac{1+A^{n}}{1-A^{n}}
$$

and thus

$$
-\sum_{\zeta^{n}=1} \frac{4 x^{2} \zeta}{(1-\zeta)^{2}+4 x^{2} \zeta}=\frac{n i x}{\sqrt{1-x^{2}}} \frac{1+A^{n}}{1-A^{n}} .
$$

Since evaluating (4.1) at $\zeta=1$ yields -1 , we subtract -1 from both sides of (4.2) to obtain

$$
\begin{aligned}
\sum_{k=1}^{\infty} 4^{k} S_{k}(n) x^{2 k} & =-\sum_{\substack{\zeta n=1 \\
\zeta \neq 1}} \frac{4 x^{2} \zeta}{(1-\zeta)^{2}+4 x^{2} \zeta} \\
& =1+\frac{n i x}{\sqrt{1-x^{2}}} \frac{1+\left(1-2 x^{2}+2 i x \sqrt{1-x^{2}}\right)^{n}}{1-\left(1-2 x^{2}+2 i x \sqrt{1-x^{2}}\right)^{n}}
\end{aligned}
$$

To see that (4.3) really is equivalent to Theorem 2.3, we make the substitution $x=\sin \theta$, which takes $A$ to $\cos 2 \theta+i \sin 2 \theta=e^{2 i \theta}$. Then (4.3) becomes

$$
\sum_{k=1}^{\infty} 4^{k} S_{k}(n) \sin ^{2 k} \theta=1+n i \tan \theta \frac{1+e^{2 i \theta n}}{1-e^{2 i \theta n}},
$$

from which the equivalence is clear.

We can apply the same approach to $\sum_{k=1}^{\infty} x^{k} \sum_{\zeta^{n}=1} R(\zeta)^{k}$ in the general case. 
THE EleCtronic JoURnAl of COMBinatorics 4 (No. 2) (1997), \#R11

Theorem 4.3. Let $R(\zeta)$ be a rational function defined for every root of unity $\zeta$. Then

$$
\sum_{k=1}^{\infty} x^{k} \sum_{\zeta^{n}=1} R(\zeta)^{k}=n T(x)+n \sum_{j} \frac{N_{j}(x)}{1-D_{j}(x)^{n}}
$$

where $T(x)$ is a rational function of the form $a x /(b+c x)$ and $N_{j}(x)$ and $D_{j}(x)$ are algebraic functions, with $D_{j}(x) \neq 0$.

Proof. Let $R(\zeta)=P(\zeta) / Q(\zeta)$, for relatively prime polynomials $P$ and $Q$. Then

$$
\sum_{k=1}^{\infty} x^{k} \sum_{\zeta^{n}=1} R(\zeta)^{k}=\sum_{\zeta^{n}=1} \frac{x R(\zeta)}{1-x R(\zeta)}=\sum_{\zeta^{n}=1} \frac{x P(\zeta)}{Q(\zeta)-x P(\zeta)}
$$

Now $Q(\zeta)-x P(\zeta)$ may be factored as $C(x) \prod_{j}\left(1-D_{j}(x) \zeta\right)$ for some algebraic functions $C(x)$ and $D_{j}(x)$, and it is not difficult to show that the $D_{j}$ must be distinct (since $Q(\zeta)-x P(\zeta)$ is irreducible over $\mathbf{C}(x)[\zeta])$. Thus $x P(\zeta) /(Q(\zeta)-x P(\zeta)$ ) has a partial fraction decomposition

$$
\frac{x P(\zeta)}{Q(\zeta)-x P(\zeta)}=T(x)+\sum_{j} \frac{N_{j}(x)}{1-D_{j}(x) \zeta}
$$

where the $N_{j}(x)$ and $D_{j}(x)$ are algebraic functions of $x$, and $T(x)$ is a rational function of $x$. More precisely $T(x)$ is 0 if the degree of $P$ is less than that of $Q$, $T(x)$ is -1 if the degree of $P$ is greater, and if $P$ and $Q$ have the same degree, with leading coefficients $p$ and $q$, then $T(x)=p x /(q-p x)$. Summing over $\zeta^{n}=1$ yields

$$
\sum_{k=1}^{\infty} x^{k} \sum_{\zeta^{n}=1} R(\zeta)^{k}=n T(x)+n \sum_{j} \frac{N_{j}(x)}{1-D_{j}(x)^{n}}
$$

A similar result holds for $\sum_{k=1}^{\infty} x^{k} \sum_{\substack{\zeta^{n}=1 \\ \zeta \neq 1}} R(\zeta)^{k}$ : the right side of (4.4) is modified by subtracting $x P(1) /(Q(1)-x P(1))$, which is always well-defined even if $R(1)$ is not. Usually when $\zeta=1$ is omitted from the sum it is because $Q(1)=0$, and in this case $x P(1) /(Q(1)-x P(1))=-1$, as in our second proof of Stanley's formula.

Finally, we give an example of a "reciprocity theorem."

Theorem 4.4. Let $m$ and $n$ be relatively prime and suppose that $0 \leq r<m+n$. Then

$$
\begin{aligned}
& \frac{1}{m} \sum_{\substack{\zeta m=1 \\
\zeta \neq 1}} \frac{\zeta^{r+1}}{\left(\zeta^{n}-1\right)(\zeta-1)}+\frac{1}{n} \sum_{\substack{\eta^{n}=1 \\
\eta \neq 1}} \frac{\eta^{r+1}}{\left(\eta^{m}-1\right)(\eta-1)} \\
& \quad=-\frac{1}{12}\left(\frac{m}{n}+\frac{n}{m}+\frac{1}{m n}\right)+\frac{1}{4}\left(\frac{1}{m}+\frac{1}{n}-1\right)+\frac{r}{2}\left(\frac{1}{m}+\frac{1}{n}-\frac{1}{m n}\right)-\frac{r^{2}}{2 m n} .
\end{aligned}
$$


Proof. First we find the partial fraction expansion of $x^{r} /\left(x^{m}-1\right)\left(x^{n}-1\right)$, which is a proper rational function since $r<m+n$. If $\zeta^{m}=1$ but $\zeta \neq 1$, then the coefficient of $1 /(x-\zeta)$ in the partial fraction expansion is

$$
\frac{\zeta^{r}}{\zeta^{n}-1} \lim _{x \rightarrow \zeta} \frac{x-\zeta}{x^{m}-1}=\frac{\zeta^{r}}{\zeta^{n}-1} \cdot \frac{1}{m \zeta^{m-1}}=\frac{\zeta^{r+1}}{m\left(\zeta^{n}-1\right)} .
$$

The coefficent of $1 /(x-\eta)$, where $\eta^{n}=1$ but $\eta \neq 1$, is computed similarly. The coefficients of $1 /(x-1)^{2}$ and $1 /(x-1)$ in the partial fraction expansion are the same as the coefficients of $1 /(x-1)^{2}$ and $1 /(x-1)$ in the expansion of $x^{r} /\left(x^{m}-1\right)\left(x^{n}-1\right)$ as a Laurent series in powers of $x-1$. To compute these coefficients, let us make the substitution $x=z+1$, so $z=x-1$. Then

$$
\frac{1}{x^{m}-1}=\frac{1}{(1+z)^{m}-1}=\frac{1}{m z+\left(\begin{array}{c}
m \\
2
\end{array}\right) z^{2}}=\frac{1}{m z}-\frac{m-1}{2 m}+\text { positive powers of } z
$$

and similarly for $1 /\left(x^{n}-1\right)$. So

$$
\begin{aligned}
\frac{x^{r}}{\left(x^{m}-1\right)\left(x^{n}-1\right)} & =(1+z)^{r}\left(\frac{1}{m z}-\frac{m-1}{2 m}+\cdots\right)\left(\frac{1}{n z}-\frac{n-1}{2 n}+\cdots\right) \\
& =\frac{1}{m n z^{2}}-\frac{m+n-2 r-2}{2 m n z}+\text { nonnegative powers of } z .
\end{aligned}
$$

(Alternatively, we can compute the needed terms of the Laurent series directly with Maple or some other computer algebra system.)

Thus the partial fraction expansion of $x^{r} /\left(x^{m}-1\right)\left(x^{n}-1\right)$ is

$$
\begin{aligned}
\frac{x^{r}}{\left(x^{m}-1\right)\left(x^{n}-1\right)}= & \frac{1}{m n(x-1)^{2}}-\frac{m+n-2 r-2}{2 m n(x-1)} \\
& +\sum_{\substack{\zeta m=1 \\
\zeta \neq 1}} \frac{\zeta^{r+1}}{m\left(\zeta^{n}-1\right)(x-\zeta)}+\sum_{\substack{\eta^{n}=1 \\
\eta \neq 1}} \frac{\eta^{r+1}}{n\left(\eta^{m}-1\right)(x-\eta)} .
\end{aligned}
$$

Now subtract the terms in negative powers of $x-1$ from both sides, and take the limit as $x \rightarrow 1$. We obtain

$$
\begin{aligned}
\frac{1}{12}\left(\frac{m}{n}+\frac{n}{m}+\frac{1}{m n}\right)+ & \frac{1}{4}\left(1-\frac{1}{m}-\frac{1}{n}\right)+\frac{r}{2}\left(\frac{1}{m n}-\frac{1}{m}-\frac{1}{n}\right)+\frac{r^{2}}{2 m n} \\
& =\sum_{\substack{\zeta^{m}=1 \\
\zeta \neq 1}} \frac{\zeta^{r+1}}{m\left(\zeta^{n}-1\right)(1-\zeta)}+\sum_{\substack{\eta^{n}=1 \\
\eta \neq 1}} \frac{\eta^{r+1}}{n\left(\eta^{m}-1\right)(1-\eta)} .
\end{aligned}
$$

The reciprocity theorem for the classical Dedekind sum is easily derived from the case $r=0$ of Theorem 4.4 see [7, Chapter 2]). Carlitz [2] has given a proof related to this one, but based on the partial fraction expansion of $(x-1) /\left(x^{m}-1\right)\left(x^{n}-1\right)$, which he derives from the Lagrange interpolation formula. See also Zagier [11] for a far-reaching generalization proved using residues (which for rational functions are equivalent to partial fraction expansion). 


\section{REFERENCES}

1. P. E. Bjørstad and H. Fettis, Asymptotic formulas from Chebyshev polynomials, Problem 6332, Solution by H.-J. Seiffert, Amer. Math. Monthly 88 (1994), 1015-1017.

2. L. Carlitz, The reciprocity formula for Dedekind sums, Pacific J. Math. 3 (1953), 523-527.

3. L. Carlitz, A degenerate Staudt-Clausen theorem, Archiv der Mathematik 7 (1956), 28-33.

4. L. Carlitz, Degenerate Stirling, Bernoulli, and Eulerian numbers, Utilitas Math. 15 (1979), $51-88$.

5. A. J. Duran, A sequence of polynomials related to roots of unity, Problem E 3339, Solution by R. J. Chapman and R. W. K. Odoni, Amer. Math. Monthly 98 (1991), 269-271.

6. F. T. Howard, Nörlund's number $B_{n}^{(n)}$, Applications of Fibonacci Numbers, Vol. 5 (G. E. Bergum, A. N. Philippou, and A. F. Horadam, eds.), Kluwer Acad. Publ., Dordrecht, 1993, pp. 355-366.

7. H. Rademacher and E. Grosswald, Dedekind Sums, Carus Mathematical Monographs, No. 16, Mathematical Association of America, 1972.

8. L. Smith, The e-invariant and finite coverings, Indiana Math. J. 24 (1975), 659-675.

9. L. Smith, personal communication, 1993.

10. R. P. Stanley, Invariants of finite groups and their applications to combinatorics, Bull. Amer. Math. Soc. (N.S.) 1 (1979), 475-511.

11. D. Zagier, Higher dimensional Dedekind sums, Math. Ann. (1973), 149-172. 